\title{
The use of iron oxide nanoparticles for pancreatic cancer therapy
}

\begin{abstract}
Over the last decade major advances have been made in the treatment of cancer such as breast and leukaemia. However, no satisfactory progress has been made in the effective treatment of pancreatic cancer. Treatment of this disease is hindered by resistance of tumor cells to chemotherapy and impaired drug delivery after administration. Pancreatic adenocarcinomas characteristically form a dense stroma, which hinders drug penetration. Increasing administration dosage may provide increased therapeutic effects. However, toxic drug molecules do not act selectively to tumor cells and, as such; a vast range of undesirable side effects can be experienced. Nano-sized formulations of cytotoxic agents have proved to passively target pancreatic adenocarcinomas and promote increased drug efficacy. This is thought to be due to the accumulation via enhanced permeability and retention resulting in deeper drug penetration. Nanoparticles with easily modified surfaces have been investigated extensively in recent years and play a pivotal role in biomedicine. In recent years, magnetic NPs have been increasingly explored for clinical applications, such as drug delivery, magnetic resonance imaging and magnetic fluid hyperthermia for diagnosis and cancer therapy. In comparison with traditional cancer therapy, magnetic field operated therapeutic approaches can treat cancer in an unconventional but more effective and safer way. In this literature review, we highlight the recent advances in the use of iron oxide nanoparticles in pancreatic cancer therapy.
\end{abstract}

Keywords: Metallic iron oxide, Drug delivery, Pancreatic cancer, Magnetic resonance imaging, Theranostic
Volume I Issue I - 2014

\author{
Maryam Malekigorji, Anthony DM Curtis, \\ Clare Hoskins \\ Institute of Science and Technology in Medicine, Keele \\ University, UK
}

\begin{abstract}
Correspondence: Clare Hoskins, Institute of Science and Technology in Medicine, Keele University, Keele, Staffordshire, UK,Tel 44I782-734799 Email c.hoskins@keele.ac.uk
\end{abstract}

Received:September 13,2014 | Published: October 01, 2014
Abbreviations:MNPs, Magnetic Iron Oxide Nanoparticles; EPR, Enhanced Permeability and Retention; MRI, Magnetic Resonance Imaging; NPs, Nanoparticles; SPION, Super Paramagnetic Iron Oxide Nanoparticles; PDAC, Pancreatic Ductal Adenocarcinoma; RES, Reticulo Endothelial System

\section{Introduction}

Cancer is described as the most hazardous class of disease categorized by uncontrolled cell growth. ${ }^{1,2}$ It is the third leading cause of death (after heart disease and stroke) in developed countries as well as the second leading cause of death (after heart disease) in the United States. Studies have demonstrated that there were 10 million new instances, about 6 million deaths and 22 million patients living with cancer worldwide in the year $2000 .^{3}$

Pancreatic ductal adenocarcinoma, known as PDAC, is the most common epithelial, exocrine pancreatic malignancy, i.e. showing for more than $80 \%$ of the malignant neoplasm of the pancreas. ${ }^{4}$ It is still the fourth most common cause of cancer-related death in the Western world. ${ }^{5}$ There is a direct correlation between pancreatic cancer diagnosis and increasing age with a peak incidence of the disease occurring in the 65-75 year age group. ${ }^{6}$ Patients with untreated metastatic pancreatic cancer normally live for 3-5 months and 6-10 months for locally advanced disease. ${ }^{7}$ The majority of cases are diagnosed in the malignant stages, making curative therapy impossible and leading to poor prognosis and incidence equalling mortality. ${ }^{8}$ In the year 2000, there were 217,000 new cases of pancreatic cancer with 213,000 deaths worldwide, while in Europe there were 60,139 new patients with 64,801 deaths. ${ }^{9}$ In the UK, 7,152 new cases were seen with 7,250 deaths as a result of PDAC. ${ }^{10}$ PDAC affects more western/ industrialized citizens than other parts of the world. The highest prevalence has been shown among Maoris in native Hawaiians, New
Zealand, and Black American societies, while people living in India and Nigeria have the lowest reported morbidity. ${ }^{11,12}$

Gemcitabine is the only chemotherapy available clinically for pancreatic cancer treatment. Unfortunately, it proves effective in as few as $23.8 \%$ of patients with the only alternative being surgical removal of the localized tumor (Figure 1) ${ }^{13-15}$ Thus, there is huge requirement to increase the efficacy of this treatment as well as exploring alternative therapies.

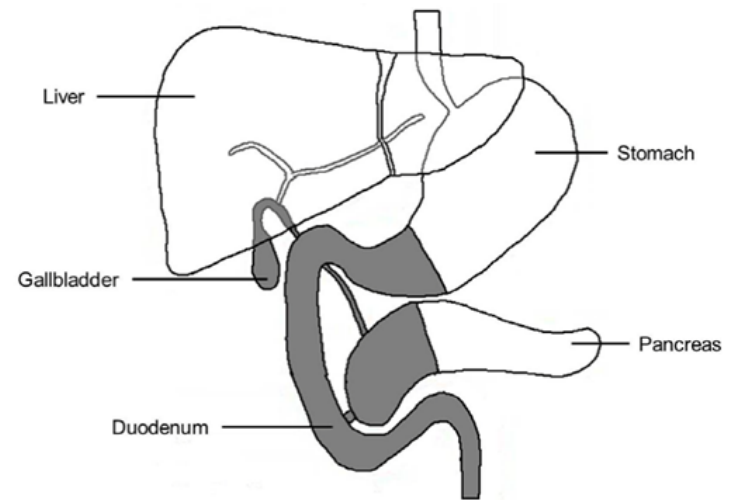

Figure I Diagram showing the total area required to be removed for a pylorus preserving pancreatic duodenectomy.

\section{Nanoparticles in biomedicine}

Nanoscience and nanotechnology has become a versatile and promising platform for creating novel materials with enhanced properties and potential applications in cancer therapy. Nanotechnology, a wide research field which includes chemistry, engineering, biology and medicine, has excellent potential for early detection, accurate diagnosis, and treatment of cancer. ${ }^{16}$ Using 
materials at nanoscale leads to a "big revolution" in healthcare and medical therapies. Nanoparticles (NPs) are usually smaller than several hundred nanometres: in comparison with large bio molecules such as antibodies, receptors and enzymes. NPs can undergo many interactions with biological molecules both on the surface of and inside the cells due to their size (one hundred to ten thousand times smaller than human cells), which may revolutionize cancer diagnosis and treatment. The most commonly studied NPs include quantum dots. ${ }^{17}$ carbon nanotubes.$^{18}$ paramagnetic NPs. ${ }^{19}$ liposomes. ${ }^{20}$ gold NPs. ${ }^{21}$ polymeric, lipid and silver NPs. ${ }^{22,23}$ Quantum dots are useful in biological labelling and detection due to their size-dependent fluorescence properties. ${ }^{24-26}$ Magnetic NPs have been used for cell sorting. ${ }^{27}$ MRI. ${ }^{28-30}$ drug delivery. ${ }^{31-33}$ and magnetic hyperthermia therapy. ${ }^{34-36}$ Lipid and polymeric NPs have been used to encapsulate therapeutic molecules to increase drug solubility, safety and delivery efficiency based on the enhanced permeability and retention (EPR) effect of the tumor tissue. ${ }^{37,38}$ Carbon-based NPs, especially carbon nanotubes, have found increasing interest from the point-of-view of biomedical applications such as photo thermal therapy. ${ }^{39}$ and drug delivery. ${ }^{40-42}$

The suspension stability (i.e. ability to remain in solution indefinitely) of NPs in physiological conditions is one of the most vital factors for their successful usage in biomedicine. This is dependent on the physical and chemical composition of the NPs, the charge in particular. When discussing NPs, size and colloidal stability (i.e. the capability of particles to resist aggregation, particularly for magnetic NPs) in physiological environments is a crucial factor to show whether a particle has the potential for clinical application. Although characterization of a particle's properties such as surface charge, surface area and crystallinity have been successively taken into account. ${ }^{43}$ an in depth understanding of the basic mechanisms including NP-protein interactions and their colloidal behaviour in different physiological environments is needed. ${ }^{44}$ The therapeutic outcome of chemotherapeutic treatments has considerably improved in the last decade but one of the main reasons for failure is correlated with the presence of multidrug resistance-associated protein in cancer cells. Once in biological media, proteins and other biological molecules quickly compete to bind onto the NP surface, leading to the formation of a protein corona (Figure 2).

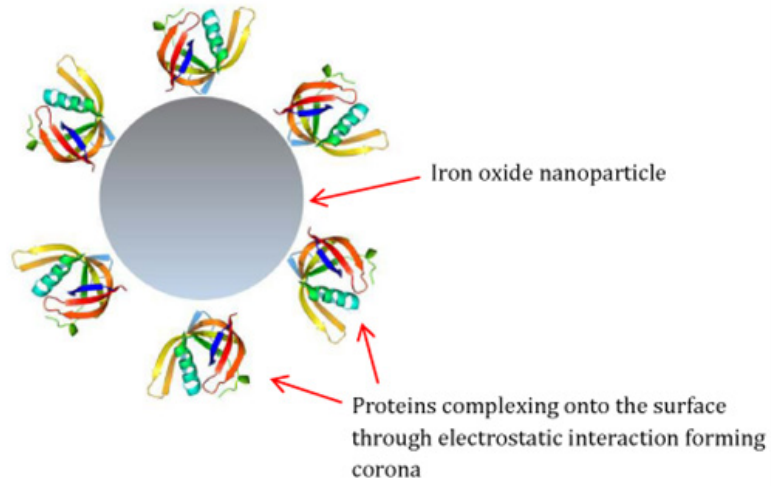

Figure 2 Protein corona formed around an iron oxide nanoparticle in biological media.

This critically defines the biological identity of the particle, whilst shielding its original surface properties. The biophysical properties of such a particle-protein complex may differ significantly from those of the formulated particle. Therefore, the biological activity and particle bio distribution are predominantly influenced by the nanoparticleprotein complexes.
Nano-sized formulations of cytotoxic agents have proved to passively target pancreatic adenocarcinomas and promote increased drug efficacy. This is thought to be due to the accumulation via EPR resulting in deeper drug penetration (Figure 3). The blood vessels supplying the blood to cancerous tissue are leaky and disorganized. The tumor tissues also experience poor lymphatic drainage. Nanoparticle size plays a significant role in their ability to extravasate from the bloodstream and reach the tumor tissue. The size of NPs used in a drug delivery system should be large enough to prevent filtration by the kidney and small enough to avoid the capture by the liver and spleen..$^{45}$ Nano-structures $(<120 \mathrm{~nm})$ are capable of entering the highly permeable blood capillaries which supply the rapidly growing tumors. ${ }^{46}$ This however does not occur in normal tissue as the blood vessels are well formed and non-porous. Once inside the capillaries, they accumulate and are retained in the tumor as a result of the poor lymphatic drainage. Additionally, formulation with appropriate nanoparticles can enhance physicochemical properties such as aqueous solubility leading to higher therapeutic efficiency.

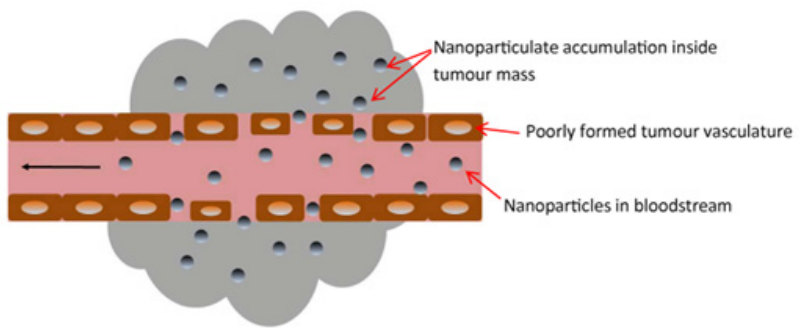

Figure 3 Schematic representation of the enhanced permeability and retention effect.

Previously, the use of nano-sized graft amphiphilic polymers (Poly(allylamine)g-cholesterol)forencapsulationanddeliveryofanovel drug for pancreatic cancer (Bisnaphthalimidopropyldiaaminooctane, BNIPDaoct) was reported. ${ }^{47}$ BNIPs are a series of novel compounds which have exhibited exciting potential as chemotherapy agents. ${ }^{47-49}$ However their clinical use is hindered by undesirable physical properties; hence they require formulation before administration. Here the polymer nano-aggregates formed were passively targeted. The formulation was administered in vivo on a pancreatic cancer model using nude tumor bearing mice over a period of 4 wks. Although the BNIPDaoct dose was eight-fold less than clinically used gemcitabine, the formulation was able to reduce tumor growth in xenograft mice with comparable results. ${ }^{47}$ This study highlights the undoubtedly promising potential of nanotechnology formulations in the delivery of drug molecules which are otherwise discarded due to their poor physicochemical properties.

However, some studies have suggested that the EPR effect alone is not sufficient for treatment of diseases such as pancreatic cancer. $^{50-52}$ This is due to poor diffusion of nanoparticles within the dense collagen matrix of the interstitial spaces and hence poor tumor penetration can occur. To overcome such issues, multistage nanoparticles which possess the ability to change size in different media have been developed. Wong et al. ${ }^{52}$ developed gelatin coated quantum dots which undergo structural degradation from $100 \mathrm{~nm}$ to $10 \mathrm{~nm}$ upon exposure to the tumor microenvironment. This proof of principle study showed how deeper tissue penetration could be achieved with the smaller nanoparticles compared with the initial 100 $\mathrm{nm}$ particles. Future studies are now concerned with incorporating nanomedicine carriers within the system in order to allow for deep penetration chemotherapy. 


\section{Metallic nanoparticles}

Over the past decade metallic NPs have been extensively studied for their potential in biomedical applications. ${ }^{53-55}$ Compared with other NPs, metallic NPs have proven to have unique chemical and physical properties based on their quantum-size which lead to a range of interesting biomedical applications. They can be easily synthesized with a high level of control of their size, shape and composition. Commonly studied metallic NPs include metal oxides such as iron oxide. ${ }^{5 \mathrm{r}}{ }^{6}$ copper oxide.$^{57}$ zinc oxide. ${ }^{58}$ and aluminium oxide. ${ }^{59}$ It is essential that metallic NPs are carefully surface engineered before introduction into biological environments due to their inherent instability and associated toxicity. ${ }^{60-62}$ The particular focus of this review is on the use of iron oxide nanostructures for pancreatic cancer.

\section{Iron oxide metallic nanoparticles}

Magnetic iron oxide nanoparticles (MNPs) have been the focus of vast scientific interest due to their potential for numerous applications in nanomedicine (Figure 4). ${ }^{63,64}$ These include being utilized in the recovery of metal ions and dyes, magnetic bio separation, targeted therapy, drug delivery, biological detection and imaging. Magnetic separation techniques possess the advantage of rapid, high efficacy, and cost-effectiveness. Also, they have been shown to be highly efficient as supports in heterogeneous catalytic reactions owing to the high specific area and magnetic recoverability. MNPs possess large surface area to volume ratios due to their nano-size, low surface charge at physiological $\mathrm{pH}$ and they aggregate easily in solution due to their inherent magnetic nature. In some cases an unwanted aggregation may decrease the long term stability of products leading to large nanoparticle clusters which are undesirable for medical application. Additionally, degradation of iron oxide into free ions in physiological environments. ${ }^{65}$ has been reported to increase free radical production in cells causing damage which may cause cell death. ${ }^{66-68}$ Therefore, these particles are commonly coated with organic macromolecules such as poly(acrylic acid) PAA..$^{6}$ Dextran.${ }^{70}$ and poly(ethyleneimine) (PEI). ${ }^{71}$ or coatings such as silica. ${ }^{72}$ carbon..$^{73}$ or precious metals (e.g. gold or silver). ${ }^{74}$ Common problems with chemically synthesized hybrid structures are the multiplicity of synthesis steps, incomplete understanding of the fundamentals of particle formation and, finally, a broad range of structures with ill-controlled configurations are produced. $^{75,76}$

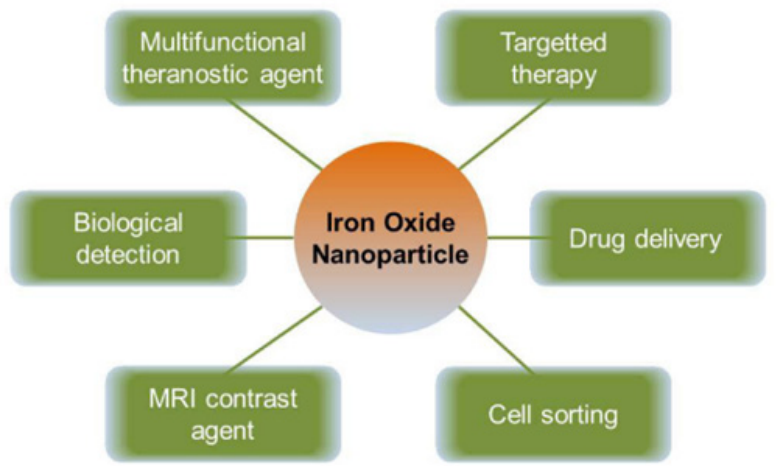

Figure 4 Schematic diagram showing uses of iron oxide nanoparticles in biomedicine.

The most desirable MNPs are composed of the iron oxide core surrounded by a biocompatible surface coating which shows stabilization under physiological environment. With further surface modification, the attachment of functional ligands and drug molecules is possible allowing for increased functionality. The modification and functionalization of MNPs have been shown to improve their magnetic properties and influence their behaviour in vivo. ${ }^{56,77}$ MNPs have been clinically exploited as contrast enhancement agents in magnetic resonance imaging (MRI). This is due to their ability to enhance the proton relaxation of specific tissue.

\section{Types of iron oxide nanoparticles}

Super paramagnetic iron oxide nanoparticles: Iron oxide nanoparticles are typically classed as super paramagnetic or ferromagnetic based on their size. Although the majority of studies in nanomedicine are based on super paramagnetic NPs, some studies have also highlighted the potential of ferromagnetic.

The term superparamagnetism is assigned to the magnetic phenomena observed in fine magnetic particle systems exhibiting close similarities to atomic paramagnetism. Freeman et al. were the first to describe the concept of the use of magnetism in medicine in the 1970s. ${ }^{31}$ Superparamagnetism occurs when the size of the nanoparticulate is so small $(20 \mathrm{~nm}$ or less) resulting in a singledomain nanoparticle whose magnetic anisotropy barrier is lower than the thermal energy, and in consequence, the orientation of the magnetic moment of the particle is unstable due to the thermal agitation, like the atomic moments in a paramagnet. Unlike their paramagnetic counterparts, super paramagnetic materials retain no remnant magnetization upon the removal of an external magnetic field. ${ }^{78,79}$ Super paramagnetic iron oxide nanoparticles (SPIONs) are small synthetic $\alpha-\mathrm{Fe}_{2} \mathrm{O}_{3}$ (hematite), $\gamma-\mathrm{Fe}_{2} \mathrm{O}_{3}$ (maghemite) or $\mathrm{Fe} 3 \mathrm{O} 4$ (magnetite) particles with a core diameter ranging from $10 \mathrm{~nm}$ to 20 $\mathrm{nm}$. SPIONs have been exploited as targeted magnetic resonance contrast agents for MRI, which improve diagnosis of progressive diseases like cancer in their early phases.$^{80}$ From a drug delivery viewpoint, targeting of cancer is the most pursued area, with emphasis on delivery of radio therapeutics and chemotherapeutics. ${ }^{81}$ However, increasing applications of SPIONs have also been used in the areas of cell death by using local hyperthermia, gene delivery, and delivery of antibodies and peptides to their site of action.

Different synthetic routes have been established in the fabrication of SPIONS ranging from physical methods such as mechanical grinding and bio mineralization processes to chemical means such as co precipitation methods, micro emulsion methods, hydrothermal syntheses, sol-gel syntheses, electrochemical methods, sonochemical reactions, polyol methods, flame-assisted methods, thermal decomposition methods, etc. By using co precipitation methods the size of the particle can be controlled by three different factors: temperature, base concentration and presence of surfactants. ${ }^{82}$ In comparison with the physical methods and the bio mineralization processes, the chemical methods, especially the solution-based synthetic ways, are generally more suitable for producing SPIONs for MRI application because they show great advantages in controlling the particle size, size distribution, degree of crystallinity and phase purity, which are the most fundamental parameters in terms of MRI applications.

There are two main pathways, through which SPIONs may be prematurely removed from circulation, either via uptake by the RES $(200 \mathrm{~nm})$ or through renal clearance mechanism (Ps $<5.5 \mathrm{~nm})$. Particles between 10 and $100 \mathrm{~nm}$ in diameter have the greatest circulation time. Their reduced surface area, in comparison with large SPIONs decreases the space accessible for adsorption of RES proteins. Moreover, they are still large enough to escape renal clearance. The chemical nature of the surface itself is also an important factor when designing stealthy NPs. For example some evidence suggest that the RES interaction of SPIONs $<40 \mathrm{~nm}$ in diameter tend to be influenced 
more by their surface properties than their size. A negatively charged surface increases the attachment of plasma proteins which leads to enhanced uptake whereas a positively charged surface can cause the SPIONs to adhere to cells in a nonspecific manner. In general, hydrophilic and neutral surface are preferred to minimize opsonisation and clearance. ${ }^{83}$

Two of the NPs are commercially available (amionSPARK $\AA$ and Feraheme $\left.{ }^{\circledR}\right)$, but have been chemically modified to achieve a specific function. ${ }^{84}$ This coating allows for addition of functional ligands, such as radioactive ions for PET imaging and fluorophores for fluorescent imaging.

Ferromagnetic iron oxide nanoparticles: Ferromagnetic iron oxide NPs offer some attractive possibilities in biomedicine. First of all, they have controllable sizes ranging from a few nanometres up to tens of nanometres, which locates them at dimensions that are smaller than or comparable to those of a virus $(20-450 \mathrm{~nm})$, a cell $(10-100 \mu \mathrm{m})$, a protein $(5-50 \mathrm{~nm})$ or a gene $(2 \mathrm{~nm}$ wide and $10-100$ $\mathrm{nm}$ long). This demonstrates that they may enter to a biological entity of interest. These nanomaterials can be coated with bio molecules for binding to or interacting with a biological entity, in order to produce a controllable addressing or tagging tool ${ }^{85}$ Moreover, these magnetic NPs can be influenced by external magnetic fields. In electromagnetic fields, energy can be transferred from the exciting fields to the nanoparticle, thus, ferromagnetic iron oxide NPs can be designed to resonantly react to a time-changing magnetic fields. Ferromagnetic nanoparticles possess stronger magnetic potential than their SPION counterparts which is attractive for external magnetic guidance and increased contrast-ability. ${ }^{86-88}$ These are made available in biomedicine due to the special physical properties of ferromagnetic iron oxide NPs. Commonly ferromagnetic iron oxide NPs are synthesised via co precipitation; like SPIONS size, shape and crystallinity can be tailored based on reaction conditions. While SPIONs are less magnetic they are often preferred due to the relative instability of ferromagnetic, making them difficult to suspend due to aggregation and making them more challenging for biomedical applications.

\section{Iron oxide for MRI}

Iron oxide NPs was used for the first time in clinic to image liver tumor and metastases. Multi-functional MNPs are currently under evaluation for use in improving the delineation of brain tumor boundaries and quantifying tumor volumes.$^{89,90}$ Many SPIONs are in the first stage of clinical trials or experimental study. ${ }^{91-95}$ and several formulations have been approved for clinical use for medical imaging and therapeutic applications. Some examples are: Lumiren ${ }^{\circledR}$ for bowel imaging. ${ }^{96}$ Feridex IV ${ }^{\circledR}$ as a liver and spleen imaging agent. ${ }^{97}$ Combidex ${ }^{\circledR}$ for lymph node metastases imaging..$^{98}$ and most recently, Ferumoxytol ${ }^{\circledR}$ for iron replacement therapy.99 Despite the great success of modern nanomedicine in creating this new class of medications, there is still a lack of complete theoretical models describing the electron structure of the complex compounds of iron oxide NPs with medical molecules.

MRI is a well-suited tool for in vivo cell tracking because of its high spatial resolution. It is due to the fact that hydrogen protons will align and process through which these protons coming back to their initial state is known as the relaxation phenomenon. Two independent processes, longitude relaxation ( $\mathrm{T} 1$ recovery) and transverse relaxation (T2 decay) are monitored to generate the MR image. Paramagnetic and super paramagnetic contrast agents change the relaxation time significantly by transferring their magnetic relaxation to the surrounding nuclei, so by selecting a specific monitoring delay the signal between contrasted and non-contrasted areas becomes significant (Figure 5). In recent years, the use of SPIONs to visualize cell migration has been used clinically, showing the potential capabilities of monitoring cellular therapies with MRI. The detection threshold for SPION-labelled cells is influenced by acquisition parameters and magnetic field strength. The drawback of MRI is that it requires high concentrations of contrast agents because it has low sensitivity. A high concentration of iron oxide increases toxicity and causes concern. However, making aggregated particles is an alternative way to increase the sensitivity. Aggregated particles have a stronger magnetic field, therefore increasing the concentration is not necessary. ${ }^{53}$ In line with the recent studies on cellular fate and toxicity of polymer coated iron oxides, most of the iron oxide nanoparticulate which were used clinically as contrast agents has been removed from use in humans in the UK. These include Feridex IV ${ }^{\circledR}$ and Luminex ${ }^{\circledR}$. As such research is now underway to use highly rigid coatings which produce biocompatible nanoparticulate which maintain the unique contrast ability of their predecessors.

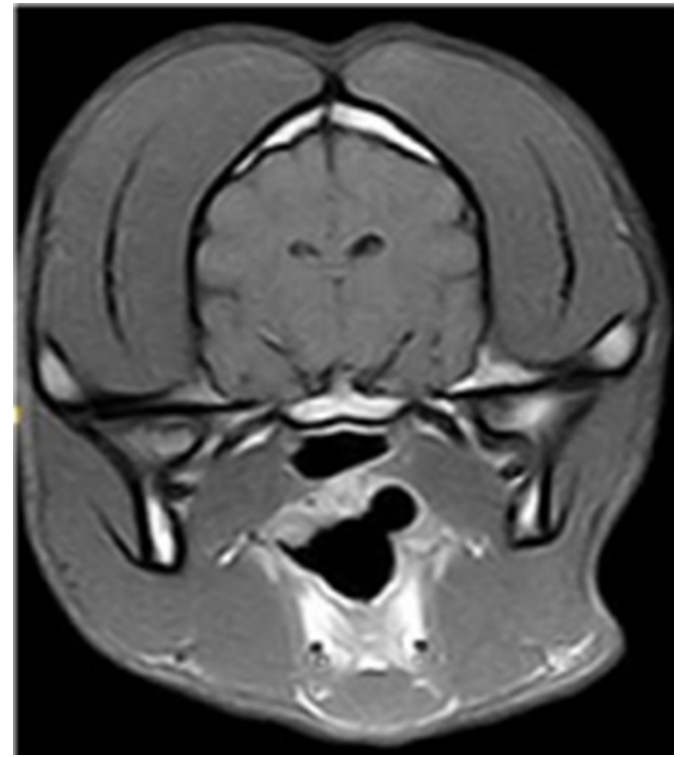

Figure 5 Typical MR image of a healthy canine brain using T2 imaging agent showing difference in contrast between various areas.

Wu and colleagues investigated MR imaging of human pancreatic cancer xenograft labelled with SPIONs in nude mice. ${ }^{100}$ Tumor xenografts were induced in nude mice by the inoculation of human pancreatic cancer cells labelled with SPIONs. The unlabelled cancer cells served as a control. MR imaging was performed with a $1.5 \mathrm{~T}$ MR scanner for the tumor xenograft at the first, second and third week after the inoculation. Wu found that the tumor xenograft was induced in $100 \%$ nude mice on MR imaging for both groups in the first week after the inoculation. In the SPION group, the tumors showed homogeneous hypo intensity on $\mathrm{T}_{1}-$ and $\mathrm{T}_{2}$-weighted and FIESTA images 1 week after inoculation. Two and 3 weeks after inoculation, the centre of the tumors was still hypo intense on all the above sequences. The tumor periphery is intense on $T_{1}$-weighted, and hyper intense on $\mathrm{T}_{2}$-weighted and FIESTA images. The tumors in the control group were homogeneously hypo intense or is intense on $\mathrm{T}_{1}$-weighted, and hyper intense on $\mathrm{T}_{2}$-weighted and FIESTA images in the first, second and third week after the inoculation. The size and signal-to-noise ratio of the tumor centre in the group inoculated with the SPIONs decreased in all $\mathrm{T}_{1}$ - and $\mathrm{T}_{2}$-weighted images and FIESTA. These results highlight that human pancreatic cancer cells labelled with SPIONs can induce tumor xenograft in nude mice and MRI can monitor the kinetics of SPIO distribution in tumor xenografts. ${ }^{100}$ 


\section{Iron oxide for magnetic hyperthermia}

Magnetic hyperthermia is renowned as an alternative treatment which could be used alone or as an adjunct to chemotherapy and/ or radiation for cancer treatment. Iron oxide NPs will generate heat when subjected to strong magnetic fields. Upon application of an electromagnetic field, the moments of the iron oxide NPs fully or partially align in the direction of the field. At a unique frequency to the NPs, the magnetic moments lag behind the field leading to a lag in the magnetic response. As such, the magnetic moments do not follow the same trajectory during the reversal of orientation of the applied magnetic field. A hysteresis loop is generated which is evident when the reversal is inhibited the associated energy loss is dissipated in the form of heat energy. In single domain materials, magnetic moment can fluctuate randomly by thermal fluctuation at high temperatures. At lower temperatures, the thermal energy is reduced resulting in a blocking of the magnetic moments. This is known as the blocking temperature and is specific to each size and shape of NP. Smaller particles occupy smaller volumes and hence possess a lower energy barrier and lower blocking temperature.

Magnetic crystal suspensions of iron oxide NP's store the energy of alternating magnetic fields and release this energy as heat causing hyperthermic stress in cancer cells. ${ }^{101}$ In targeted magnetic hyperthermia treatment of cancers, MNPs act as thermal seeds under an alternating magnetic field (Figure 6) ${ }^{86-88}$ Increasing temperature above $40^{\circ} \mathrm{C}$ improves the radiation effect and causes thermoablation of the cancer cells. In one interesting study Kossatz and co-workers investigated the therapeutic effects of magnetic hyperthermia induced by super paramagnetic iron oxide nanoparticles on pancreatic cancer (BxPC-3) xenografts in mice in vivo. The SPIONs were injected intratumorally into the pancreatic model followed by cumulative heating to $43^{\circ} \mathrm{C}$. Histological analysis of magnetic hyperthermia treated tumor tissue exhibited alterations in cell viability (apoptosis and necrosis) and showed a decreased cell proliferation compared with the controls. This highlights the use of magnetic hyperthermia in pancreatic cancer therapy. ${ }^{102}$

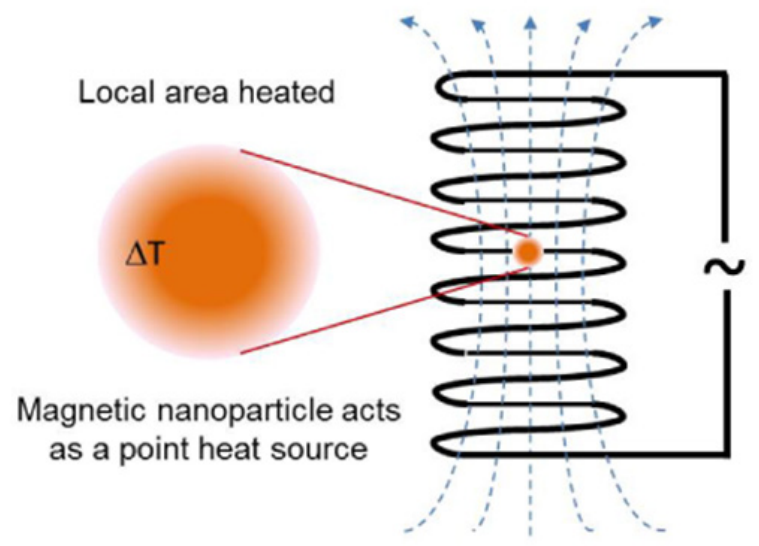

Figure 6 Schematic of mechanism of hyperthermia induction inside magnetic field.

In another study Basel loaded MNPs into mouse monocytemacrophage like (RAW264.7) cells. These cells are renowned for their tumor homing activity. A murine xenograft model of disseminated peritoneal pancreatic cancer was then generated by intraperitoneal injection of Pan-02 cells. After tumor development, monocyte/macrophage-like cells loaded with MNPs were injected intraperitoneally and allowed to migrate into the tumor. After three days the mice were placed in an alternating magnetic field for 20 min to initiate hyperthermia. This treatment regimen was repeated three times. A survival study demonstrated that this technology significantly increased survival in this murine pancreatic cancer model. The average post-tumor insertion life expectancy increased to $31 \%$. It was concluded that this system has great potential to become a useful method for specifically and actively delivering nanoparticles for local hyperthermia treatment of cancer. ${ }^{103}$

One drawback to the current state of this technology is the inconsistencies between experimental results and predictions of the amount of heat generated by the MNPs based on the existing simple models, and this has been a major disadvantage to optimize the design of magnetic particles for practical application. ${ }^{104}$

\section{Iron oxide nanoparticles as vehicles for chemotherapy}

Iron oxide NPs are increasingly being investigated for their potential as drug delivery vehicles for chemotherapy. ${ }^{105-107}$ Nanosized formulations of cytotoxic agents have proved to passively target pancreatic adenocarcinomas and promote increased drug efficacy. ${ }^{47}$ This is thought to be due to the accumulation via EPR resulting in deeper drug penetration. This factor combined with the rapid diagnostic and treatment platform of this technology results in a system with great potential to act as a localised therapy with reduced dosages, thus minimising harsh side effects and improve clinical outcomes for patients with pancreatic cancer.

Many chemical methods have been applied for the conjugation of therapeutic, targeting and imaging carrier molecules with NP surfaces. These can be classified into covalent linkage strategies (direct nanoparticle conjugation, covalent linker chemistry, click chemistry) and physical interactions (hydrophilic/hydrophobic, electrostatic and affinity interactions). Physicochemical properties, functional groups found on the NPs coating and ligands are factors which influence the choice of chemistry. The primitive aim is to bind the targeting, therapeutic or imaging moiety without compromising its functionality. Functionality in such assemblies is dictated by the nature of the ligand (e.g. conformation of bio molecules) and the manner in which it is attached. For instance, if an antibody is conjugated to the NP but its recognition site is shielded by proteins, it might lose its potency to bind or reach to a target.

The surface of iron oxide NPs has been modified with anticancer drugs such as doxorubicin (Dox). ${ }^{108}$ Catechin-Dextran. ${ }^{109}$ and Paclitaxel. ${ }^{108}$ Catechin-Dextran conjugated Endorem NPs increased the intracellular concentration of the drug compared with the free drug. Endorem is an FDA approved dextran coated iron oxide NP. The Catechin-Dextran-Endorem formulation induced apoptosis in $98 \%$ of human pancreatic cancer cell line (MIA PaCa-2) placed under a magnetic field. The findings suggest that conjugation of catechindextran with Endorem enhances the anticancer activity of this drug and provides a novel means for targeted drug delivery to tumor cells driven by magnetic fields. The authors concluded that the ability to spatially control the delivery of the catechin-dextran by a magnetic field makes it a promising agent for cancer therapy. ${ }^{108}$

Often the surface of iron oxide NPs for chemotherapy will also contain a targeting agent which can identify the receptors overexpressed on the external surface of cancer cells. ${ }^{110}$ Recently Lee et al..${ }^{11}$ designed urokinase plasminogen activator receptor (uPAR)-targeted magnetic iron oxide nanoparticles (IONPs) carrying gemcitabine as a chemotherapy drug for targeted delivery into uPAR-expressing tumor and stromal cells. The novel formulation was prepared by conjugating IONPs with the amino-terminal fragment peptide of the receptorbinding domain of UPA. UPA is a naturally occurring ligand of UPAR, 
and gemcitabine via a lysosomally cleavable tetrapeptide linker. These theranostic nanoparticles were designed to enable intracellular release of gemcitabine following receptor-mediated endocytosis into tumor cells whilst providing contrast enhancement in magnetic resonance imaging (MRI) of tumors the results demonstrated the $\mathrm{pH}$ and lysosomal enzyme-dependent release of gemcitabine, preventing the drug from enzymatic degradation whilst imaging inside the tumor was possible.

\section{Gold coated iron oxide nanoparticles}

In order for these MNP's to be safe they are coated with materials such as silica, polymers or gold. Iron oxide NPs coated with gold are referred to as hybrid nanoparticle HNPs, (Figure 7). Gold surfaces bind strongly with thiol (-SH) containing molecules via dative covalent linkages. This allows for ease of surface modification. Some studies have exploited this for the treatment of pancreatic cancer. Recently, Barnett and colleagues published a study on the attachment of 6-thioguanine onto HNPs. ${ }^{12}$ 6-TG is an antitumor agent used clinically for the treatment of leukaemia. ${ }^{113} 6$-TG belongs to the thiopurine series of compounds which are renowned for their immunosuppressant properties and are commonly used in organ transplants to reduce rejection of new tissue and also in the treatment of Crohn's disease. ${ }^{114}$ In this study, 6-TG was successfully conjugated onto the HNP surface with a ratio of 3:1:10 Fe: Au: 6-TG (wt: wt: wt). After incubation with BxPC-3 cells, enhanced cellular uptake was reported with the 6-TG-conjugated HNPs compared with free drug. A 10 -fold decrease in IC50 was also observed highlighting the exciting potential of HNPs for use as drug carriers. Physical conjugation of other anticancer agents such as cisplatin onto HNP surfaces has also been reported. ${ }^{115}$

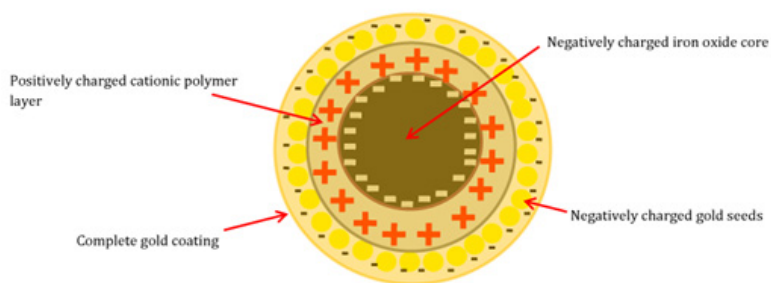

Figure 7 Schematic diagram of gold coated iron oxide nanoparticle showing electrostatic charges within each layer.

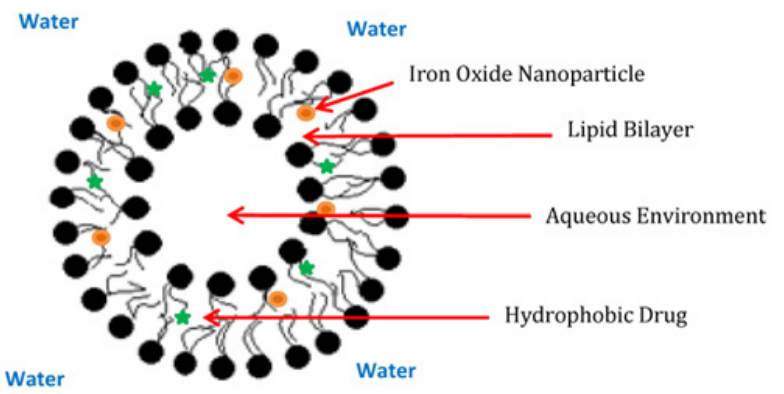

Figure 8 Schematic diagram of a magnetic-liposome encapsulating a hydrophobic drug.

HNPs possess dual functionality. As well as retaining the inherent magnetic properties of the iron oxide core they also gain optical properties arising from the gold shell. Colloidal gold possesses unique surface plasmon resonance (SPR) properties. When nanoparticles are irradiated with light of appropriate wavelength, particles undergo absorption and scattering of the photons. This absorption of gold nanoparticles is followed by rapid conversion of light into thermal energy. ${ }^{116}$ This unique property can be exploited for further applications such as photo thermal ablation and thermoresponsive drug delivery. ${ }^{108}$ Clinically, the optimal wavelengths for laser irradiation of gold nanoparticles are within the 'biological near infrared region (NIR)'. ${ }^{117-119}$ Laser beams inside the NIR window are capable of deep tissue penetration due to the high transmisivity of water and haemoglobin within these wavelengths. ${ }^{120}$

Recently, Guo and colleagues have reported the photo thermal ablation of human pancreatic cell line (PANC-1). Here they showed that GoldMag ${ }^{\circledR}(50 \mu \mathrm{gmL}-1)$ nanoparticles were capable of cellular internalisation and temperature elevation after near infrared irradiation (7.9 Wcm-1, $5 \mathrm{~min}$ ). The temperature elevation achieved was as high as $79.51^{\circ} \mathrm{C}$ which resulted in only $2.3 \%$ cellular proliferation after $24 \mathrm{~h}$ compared with $47.0 \%$ in the non-irradiated control. ${ }^{121}$ Use of HNPs for thermo-responsive drug delivery is now a major focus. This technology benefits from magnetic properties reduced toxicities and the ability to target the drug release using focussed laser irradiation. Due to the relative age of this technology to the best of our knowledge no work has yet been published for pancreatic cancer therapy.

\section{Iron oxide incorporated into supramolecular systems}

As scientific knowledge increases more sophisticated platforms are emerging. Iron oxide NPs and HNPs have been incorporated into large macromolecular structures to exploit their magnetic potential whilst maintaining the properties of the larger system. One example of this is the fabrication of magneto-liposomes. Here iron oxide is incorporated into the liposome structure (Figure 8). Liposomes are spherical structures composed of a phospholipid bilayer surrounding aqueous reservoir. ${ }^{121,122}$ Liposome vesicles are composed of unilamellar or multilamellar lipid bilayer which have alternative aqueous layers sandwiched between the bilayer. ${ }^{123}$ Frascione and colleagues demonstrated the successful incorporation of super paramagnetic iron oxide NPs into a PEG coated liposome. The particle diameter of magnetic liposomes ranged between 100-200 nm. An effective iron oxide loading was achieved, with encapsulation efficiency between $74 \%$ and $92 \%$. Their study showed that even after incorporation into larger macromolecular systems the intrinsic magnetic nature of the SPIONs was still capable of giving good contrast-ability on the MRI.

Hydrophilic or hydrophobic drugs can become encapsulated inside the aqueous or lipid phase of the liposomes, respectively. ${ }^{122,124}$ Liposomes have been shown to improve the therapeutic efficacy of pharmaceutical drugs including ibuprofen, amphotericin B and doxorubicin. ${ }^{122,125-129}$ Incorporation of iron oxide nanoparticles into the intrinsic liposome structure results in a delivery system capable of real time tracking in vivo. Deng and colleagues fabricated a magnetic liposome structure for pancreatic cancer therapy. ${ }^{130}$ Here, ultra-small SPIONs were incorporated into a liposome containing doxorubicin forming a theranostic agent. The surface of the liposome was coated with anti-mesothelin antibodies which are over expressed in pancreatic cancer cells and subsequently poly(ethylene glycol) to give stealth properties. The novel formulation was tested on PANC-1 cells. The in vitro studies showed the magnetic liposome's possessed good relaxivity on the MRI leading to high contrastability. The in vivo antitumor study demonstrated that compared with the free drug and the liposomal formulations both with and without SPION incorporation possessed a tumor retardation effect. A tissue distribution assay further proved that magnetic liposomes were capable of selectively accumulating inside the tumor xenograft. These results indicated that the magnetic liposomes not only retained the inherent MRI capability of SPIONs well, but also they significantly improved the distribution of the SPIONs and therapeutic agents in pancreatic tumor tissues. ${ }^{130}$ 
A recent study showed successful incorporation of HNPs into the intrinsic structure of a graft amphiphilic polymer derivative of poly(allylamine) (PAA). ${ }^{131}$ Amphiphilic polymers are comprised of hydrophilic and hydrophobic domains which spontaneously aggregate into core-shell nano-aggregates in aqueous environments. Poly(allylamine) grafted amphiphiles have been reported in the solubilisation of lipophilic drug molecules including propofol, prednisolone, griseofulvin (132) and the novel anticancer therapeutic BNIPDaoct (previously discussed). ${ }^{47}$ The formation of a polymerHNP conjugate resulted in a bifunctional platform that can be used for image guided drug delivery via exploitation of the inherent magnetism possessed by the iron oxide core. Here the gold coating of the HNP formed strong dative covalent bonds with the hydrophobic oxadiazole $(\mathrm{Ox})$ pendant group via its thiol (-SH) functionality. The PAA-Ox5 aggregates exploited as drug carriers using both direct conjugation of drug molecules (6-Thioguanine, 6-TG) and encapsulation of hydrophobic drug molecules (novel anticancer agent bisnapthalamidopropyldiaminooctane, BNIPDaoct). In vitro assays carried out using human pancreatic adenocarcinoma (BxPC-3) cells showed that treatment with the polymer-HNP formulation (with both directly conjugated and encapsulated drugs) resulted in an increased drug uptake compared with the free drug. A significant decrease in the IC50 value compared with the free drug was also observed. These observations show that not only is the incorporation of HNPs feasible into amphiphilic polymers but also it can increase their drug solubilisation efficiency whilst adding imaging capability. ${ }^{131}$

\section{Discussion}

The studies highlighted show the great potential of iron oxide to be utilised in this field. More work needs to be carried out in order to determine to optimise parameters and understanding in areas such as penetration depth, drug efficacy and clearance mechanism of the nanoparticle systems. Long term stability needs to be studied in depth in order to avoid the degradation of iron oxide into free radicals and magnetic clustering causing formulation challenges. The wealth of expertise in this area is likely to overcome these issues resulting in treatment progressing through trials and into the clinic. It is imperative that more efforts focus on pancreatic cancer applications and the advantages gained over using the ranostics in cancer. In pancreatic cancer the time between diagnosis and treatment often is of detriment to the patient. Rapid diagnosis and treatment using iron oxide the ranostics may hold the key to improved clinical outcomes and patient quality of life.

\section{Conclusion}

Iron oxide has shown to hold potential as an imaging and chemotherapy for pancreatic cancer. As scientific focus increases into pancreatic cancer, the development of theranostic agents may hold the key to improved treatment of this fatal disease.

\section{Acknowledgments}

None.

\section{Conflict of interest}

None.

\section{References}

1. Chow AY. Cell cycle control by oncogenes and tumor suppressors driving the transformation of normal cells into cancerous cells. Nature Education. 2010;3(9):7.
2. Suriamoorthy P, Zhang X, Hao G, et al. Folic acid-CdTe quantum dot conjugates and their applications for cancer cell targeting. Cancer Nanotechnology. 2010;1(1-6):19-28.

3. Parkin DM. Global cancer statistics in the year 2000. Lancet Oncol. 2001;2(9):533-543.

4. Alexakis N, Halloran C, Raraty M, et al. Current standards of surgery for pancreatic cancer. Br J Surg. 2004;91(11):1410-1427.

5. Jemal A, Siegel R, Ward E, Murray T, Xu J, et al. Cancer statistics. Cancer J Clin. 2007;57(1):43-66.

6. Lowenfels $\mathrm{AB}$, Maisonneuve $\mathrm{P}$ Epidemiology and risk factors for pancreatic cancer. Best Pract Res Clin Gastroenterol. 2006;20(2):197-209.7.

7. O’Sullivan A, Kocher HM Pancreatic cancer. Br Med J Clin Evid. 2007;11:409-437.

8. Li D, Xie K, Wolff R, Abbruzzese JL Pancreatic cancer. Lancet. 2004;363(9414):1049-1057.

9. Parkin DM, Bray FI, Devesa SS Cancer burden in the year 2000. The global picture. Eur J Cancer. 2001;37(Suppl 8):S4-S66.

10. Ghaneh P, Costello E, Neoptolemos JP Biology and management of pancreatic cancer. Gut 2007;56(8):1134-1152.

11. Boyle P, Hsieh CC, Maisonneuve P et al. Epidemiology of pancreas cancer (1988). Int J Pancreatol. 1989;5(4):327-346.

12. Mack TM, Peters JM, Yu MC et al. Pancreas cancer is unrelated to the workplace in Los Angeles. Am J Ind Med. 1985;7(3):253-266.

13. Cunha Sa A, Rault A, Laurent $\mathrm{C}$ et al. Surgical resection after radiochemotherapy in patients with unresectable adenocarcinoma of the pancreas. J Am Coll Surg. 2005;201(3):359-365.

14. Jemal A, Thomas A, Murray T, Thun M Cancer statistics 2002. CA Cancer J Clin. 2002;52(1):23-47.

15. Sarkar FH, Li Y, Wang Z, Kong D Pancreatic cancer stem cells and EMT in drug resistance and metastasis. Minerva Chir. 2009;64(5):489-500.

16. Cai W, Chen X Nanoplatforms for targeted molecular imaging in living subjects. Small. 2007;3(11):1840-1854.

17. Cai W, Hsu AR, Li ZB, Chen XAre quantum dots ready for in vivo imaging in human subjects? Nanoscale Res Lett. 2007;12(6):265-281.

18. Taghdisi SM, Lavaee P, Ramezani M et al. Reversible targeting and controlled release delivery of daunorubicin cancer cells by aptamerwrapped carbon nanotubes. Eur JPharm Biopharm. 2011;77(2):200-226.

19. Thorek DL, Chen AK, Czupryna J, Tsourkas A Super paramagnetic iron oxide nanoparticle probes for molecular imaging. Ann Biomed Eng. 2006;34(1):23-38

20. Dalla Pozza E, Lerda C, Costanzo C, Donadelli M et al. Targeting gemcitabine containing liposomes to CD44 expressing pancreatic adenocarcinoma cells causes an increase in the antitumor activity. Biochim Biophys Acta 2013;1828(5):1396-1404.

21. Huang X, Jain PK, El-Sayed IH, El-Sayed MA Gold nanoparticles: interesting optical properties and recent applications in cancer diagnostics and therapy. Nanomedicine (Lond). 2007;2(5):681-693.

22. Ferrari M Cancer nanotechnology: opportunities and challenges. Nat Rev Cancer. 2005;5(3):161-171.

23. Grodzinski P, Silver M, Molnar LK Nanotechnology for cancer diagnostics: promises and challenges. Expert Rev Mol Diagn. 2006;6(3):307-318.

24. Bruchez Jr M, Moronne M, Gin P et al. Semiconductor nanocrystals as fluorescent biological labels. Science. 1998;281(5385):2013-2016.

25. Chan WC, Nie S Quantum dot bio conjugates for ultrasensitive nonisotopic detection. Science. 1998;281(5385):2016-2018. 
26. Lee KH, Galloway JF, Park J et al. Quantitative molecular profiling of biomarkers for pancreatic cancer with functionalized quantum dots. Nanomedicine. 2012;8(7):1043-1051.

27. Liberti PA, Rao CG, Terstappen LWMM Optimization of ferrofluids and protocols for the enrichment of breast tumor cells in blood. J Magn Mater. 2001;225(1-2):301-307.

28. Lawaczeck R, Bauer H, Frenzel T et al. Magnetic iron oxide particles coated with carboxydextran for parenteral administration and liver contrasting. Acta Radiol. 1997;38(4 Pt 1):584-597.

29. Meyers PH, Cronic F, Nice CM Jr. Experimental approach in the use and magnetic control of metallic iron particles in the lymphatic and vascular system of dogs as a contrast and isotopic agent. Am J Roentgenol Radium Ther Nucl Med. 1963;90:1068-1977.

30. Schleich N, Po C, Jacobs D, et al. Comparison of active, passive and magnetic targeting to tumors of multifunctional paclitaxel/SPIOloaded nanoparticles for tumor imaging and therapy. J Control Release. 2014;194:82-91.

31. Freeman MW, Arrott A, Watson JHL. Magnetism in medicine. J Appl Phys. 1960;31:S404.

32. Joubert JC, Quim AN. Magnetic Microcomposites as Vectors for Bioactive. Agents: The State of Art. Int Ed:1997; 93 S70.

33. Goodwin S, Peterson C, Hoh C, Bittner C. Targeting and retention of magnetic targeted carriers (MTCs) enhancing intra-arterial chemotherapy. J Magn Magn Mater. 1999;194(1-3):132-139.

34. Andra W, d'Ambly CG, Hergt R, et al. Temperature distribution as function of time around a small spherical heat source of local magnetic hyperthermia. J Magn Magn Mater. 1999;194(1-3): 197-203.

35. Hilger I, Hergt R, Kaiser WA. Effects of magnetic thermoablation in muscle tissue using iron oxide particles: an in vitro study. Invest Radiol. 2000;35(3):170-179.

36. Kruse AM, Meenach SA, Anderson KW, et al. Synthesis and characterization of CREKA-conjugated iron oxide nanoparticles for hyperthermia applications. Acta Biomater. 2014;10(6):2622-2629.

37. Moses MA, Brem H, Langer R. Advancing the field of drug delivery: taking aim at cancer. Cancer Cell. 2003;4(5):337-341.

38. Farokhzad OC, Langer R. Developing smarter therapeutic and diagnostic modalities. Adv Drug Deliv Rev. 2006;58(14):1456-1459.

39. Kam NW, O'Connell M, Wisdom JA, et al. Carbon nanotubes as multifunctional biological transporters and near infrared agents for selective cancer cell destruction. Proc Natl Acad Sci USA. 2005;102(33):11600-11605.

40. Bianco A, Kostarelos K, Partidos DC, et al. Biomedical applications of functionalized carbon nanotubes. Chem Commun (Camb). 2005;5:571-577.

41. Bekyarova E, Ni Y, Malarkey EB, et al. Applications of carbon nanotubes in biotechnology and biomedicine. J Biomed Nanotechnol. 2005;1(1):3-17.

42. Lin Y, Taylor S, Li H, et al. Advances toward bio applications of carbon nanotubes. J Mater Chem. 2004;14(4):527-541.

43. Rivera Gil P, Oberdorster G, Elder A, et al. Correlating physicochemica with toxicological properties of nanoparticles: the present and the future. ACS Nano. 2010;4(10):5527-5531.

44. Warheit DB. How meaningful are the results of nanotoxicity studies in the absence of adequate material characterization? Toxicol Sci. 2008;101(2):183-185.

45. Cho K, Wang X, Nie S, et al. Therapeutic nanoparticles for drug deliver in cancer. Clin Cancer Res. 2008;14(5):1310-1316.

46. Barenholz Y. Doxil ${ }^{\circledR}$ - the first FDA approved nanodrug: lessons learned. J Control Release. 2012;160(2):117-134.
47. Hoskins C, Ouaissi M, Lima SC, et al. In vitro and in vivo anticancer activity of a novel nano-sized formulation based on self-assembling polymers against pancreatic cancer. Pharm Res. 2010;27(12):2694-2703.

48. Brana MF, Ramos A. Naphthalimides as anti-cancer agents: synthesis and biological activity. Curr Med Chem Anticancer Agents. 2001;1(3):237-255.

49. Lin PK, Pavlov VA. The synthesis and In vitro cytotoxic studies of novel bis-naphthalimidopropyl polyamine derivatives. Bioorg Med Chem Lett. 2000;10(14):1609-1612.

50. Netti PA, Berk DA, Swartz MA, et al. Role of extracellular matrix assembly in interstitial transport in solid tumors. Cancer Res. 2000;60(9):2497-2503.

51. Jain RK. Delivery of molcular and cellular medicine to solid tumors. Adv Drug Deliv Rev. 2012;64(Suppl):353-365.

52. Wong C, Styliano poulos T, Cui J, et al. Multistage nanoparticle delivery system for deep penetration into tumor tissue. Proc Natl Acad Sci USA. 2011;108(6):2426-2431.

53. Bystrejewski M, Cudzilo S, Huczko A, et al. Carbon encapsulated magnetic nanoparticles for biomedical applications: thermal stability studies. Biomol Eng. 2007;24(5):555-558.

54. Thanh NTK, Green L. Functionalization of nanoparticles for biomedical applications. Nano Today. 2010;5:213-230.

55. Fadeel B, Garcia-Bennett AE. Better safe than sorry: understanding the toxicological properties of inorganic nanoparticles manufactured for biomedical applications. Adv Drug Deliv Rev. 2010;62(3):362-374.

56. Gupta AK, Gupta M. Synthesis and surface engineering of iron oxide nanoparticles for biomedical applications. Biomaterials. 2005;26(18):3995-4021.

57. Ren G, Hu D, Cheng EW, et al. Characterisation of copper oxide nanoparticles for antimicrobial applications. Int $J$ Antimicrob Agents. 2009;33(6):587-590.

58. Ansari SA, Hussain Q, Qayyum S, et al. Designing and surface modification of zinc oxide nanoparticles for biomedical applications. Food Chem Toxicol. 2011;49(9):2107-2115.

59. Ansari SA, Hussain Q. Immobilization of kluyveromyces lactis b galactosidase on concanavalin A layered aluminium oxide nanoparticles - its future aspects in biosensor applications. $J$ Mol Catal B Enzymatic. 2011;70(3-4):119-126.

60. Belyanskaya L, Manser P, Spohn P, et al. The reliability and limits of the MTT reduction assay for carbon nanotubes-cell interaction. Carbon. 2007;45(13):2643-2648

61. Fisichella M, Dabboue H, Bhattacharyya S, et al. Mesoporous silica nanoparticles enhance MTT formazan exocytosis in HeLa cells and astrocytes. Toxicol In Vitro. 2009;23(4):697-703.

62. Hafeli UO, Riffle JS, Harris-Shekhawat L, et al. Cell uptake and in vitro toxicity of magnetic nanoparticles suitable for drug delivery. $\mathrm{Mol}$ Pharm. 2009;6(5):1417-1428.

63. Acar HYC, Garaas RS, Syud F, et al. Super paramagnetic nanoparticles stabilized by polymerized PEGylated coatings. J Magn Magn Mater. 2005;293(1):1-7.

64. Neuberger T, Schopf B, Hofmann H, et al. Super paramagnetic nanoparticles for biomedical applications: possibilities and limitations of a new drug delivery system. J Magn Magn Mater. 2005;293(1):483-496.

65. Shubayev VI, Pisanic TR, Jin S. Magnetic nanoparticles for theragnostics. Adv Drug Deliv Rev. 2009;61(6):467-477.

66. Minotti G, Aust SD. The requirement for Iron (III) in the initiation of lipid peroxidation by Iron (II) and hydrogen peroxide. J Biol Chem. 1987;262(3):1098-1104. 
67. Hoskins C, Wang L, Cheng WP, et al. Dilemmas in the reliable estimation of the in-vitro cell viability in magnetic nanoparticle engineering: which tests and what protocols? Nanoscale Res Lett. 2012;7:77.

68. Hoskins C, Cuschieri A, Wang L. Cytotoxicity of polycationic iron oxide nanoparticles: Common endpoint assays and alternative approaches for improved understanding of cellular response mechanism. J Nanobiotechnology. 2012;10:15

69. Mak SY, Chen DH. Binding and sulfonation of poly(acrylic acid) on iron oxide nanoparticles: a novel, magnetic, strong acid cation nano-adsorbent. Macromolecular Rapid Communications. 2005;26(19):1567-1571.

70. Li L, Mak KY, Shi J, et al. Sterilization on dextran-coatted iron oxide nanoparticles: Effects of autoclaving, filtration UV irradiation, and ethanol treatment. Microelectronic Engineering. 2013;111:310-313.

71. Wang X, Zhou L, Ma Y, et al. Control of aggregation size of polyethyleneimine-coated magnetic nanoparticles for magnetofection. Nano Res. 2009;2:365-372.

72. Santra S, Tapec C, Theodoropoulou N, et al. Synthesis and characterization of silicacoated iron oxide nanoparticles in microemulsion: the effect of non-ionic surfactants. Langmuir. 2001;17(10):2900-2906.

73. Mendes RG, Koch B, Bachmatiuk A, et al. Synthesis and toxicity characterization of carbon coated iron oxide nanoparticles with highly defined size distributions. Biochim Biophys Acta: General Subjects. 2014;1840(1):160-169.

74. Mandal M, Kundu S, Ghosh SK, et al. Magnetite nanoparticles with tunable gold or silver shell. J Colloid Interface Sci. 2005;286(1):187-194.

75. Prabhu VM, Hudson SD. Nanoparticle assembly: DNA provides control. Nat Mater. 2009;8(5):365-366.

76. Chen W, Bian A, Agarwal A, et al. Nanoparticle superstructures made by polymerase chain reaction: collective interactions of nanoparticles and a new principle for chiral materials. Nano Lett. 2009;9(5):2153-2159.

77. Tartaj P, Morales MD, Veintemillas-Verdaguer S, Gonzalez-Carreno $\mathrm{T}$, et al. The preparation of magnetic nanoparticles for applications in biomedicine. J Phys D-Appl Phys. 2003;36(13):182-197.

78. Dunlop DJ. Super paramagnetic and single-domain threshold sizes in magnetite. J Geophys Res. 1973;78(11):1780-1793.

79. Bate G. Recording Materials Chapter 7, in Ferromagnetic Materials. Wohlfarth, Elsevier, North Holland. 1980;2:381-507.

80. Amstad E, Zurcher S, Mashaghi A, et al. Surface functionalization of single superparamagnetic iron oxide nanoparticles for targeted magnetic resonance imaging. Small. 2009;5(11):1334-1342.

81. Moghimi SM, Hunter AC, Murray JC. Long-circulating and target-specific nanoparticles: theory to practice. Pharmacol Rev. 2001;53(2):283-318.

82. Levy L, Sahoo Y, Kim KS, et al. Nanochemistry: synthesis and characterization of multifunctional nanoclinics for biological application. Chem Mater. 2002;14:3715-3721.

83. Koseoglu Y, Y1ld1z F, Kim DK, et al. EPR studies on Na-oleate coated $\mathrm{Fe} 3 \mathrm{O} 4$ nanoparticles, Second Seeheim. Conference on Magnetism. 2004;1(12):3511-3515.

84. Chen S. Polymer coated iron oxide nanoparticle for biomedical imaging. Massachusetts Institute of Technology. 2010.

85. Pankhurst QA, Connolly J, Jones SK, et al. Applications of magnetic nanoparticles in biomedicine. J Phys D: Appl Phys. 2003;36:167-181.

86. 83. Gneveckow U, et al. Magnetic force nanotherapy Biomed. Tech 2005;50:92-93.

87. Hilger I, Hergt R, Kaiser WA. Use of magnetic nanoparticle heating in the treatment of breast cancer. IEE Proc Nanobiotechnol. 2005; 152(1):33-39.
88. Hergt R, Dutz S, Muller R, et al. Magnetic particle hyperthermia: nanoparticle magnetism and materials development for cancer therapy. J Phys: Condens Matter. 2006;18:S2919-S2934.

89. Enochs WS, Harsh G, Hochberg F, et al. Improved delineation of human brain tumors on MR images using a long-circulating, super paramagnetic iron oxide agent. J Magn Reson Imaging. 1999;9(2):228-232.

90. Neuwelt EA, Varallyay P, Bago AG, et al. Imaging of iron oxide nanoparticles by MR and light microscopy in patients with malignant brain tumors. Neuropathol Appl Neurobiol. 2004;30(5):456-471.

91. Laurent S, Forge D, Port M, et al. Magnetic iron oxide nanoparticles: Synthesis, stabilization, vectorization, physicochemicalcharacterizations, and biological applications. Chem Rev. 2008;108(6):2064-2110.

92. Carthy JR, Weissleder R. Multifunctional magnetic nanoparticles for targeted imaging and therapy. Adv Drug Deliv Rev. 2008;60(11):1241-1251

93. Vu-Quang $\mathrm{H}$, Muthiah $\mathrm{M}$, Lee $\mathrm{HJ}$, et al. Immune cell-specific delivery of beta-glucan-coated inron oxide nanoparticles for diagnosing liver metastasis by MR imaging. Carbohydrate Polymers. 2012;87(2):1159-1168.

94. Wang X, Xing X, Zhang B, et al. Surface engineering antifouling optomagnetic SPIONs for bimodal targeted imaging of pancreatic cancer cells. Int J Nanomedicine. 2014;9:1601-1615.

95. Wang P, Yoo B, Yang J, et al. GLP-1R-targeting magnetic nanoparticles for pancreatic islet imaging. Diabetes. 2014;63(5):1465-1474.

96. Wang YX, Hussain SM, Krestin GP. Super paramagnetic iron oxide contrast agents: physicochemical characteristics and applications in MR imaging. Eur Radiol. 2001;11(11):2319-2331.

97. Bonnemain B. Super paramagnetic agents in magnetic resonance imaging: physicochemical characteristics and clinical applications - A review. J Drug Target. 1998;6(3):167-174.

98. Harisinghani MG, Barentsz J, Hahn PF, et al. Non-invasive detection of clinically occult lymph-node metastases in prostate cancer. $N$ Engl J Med. 2003;348(25):2491-2499.

99. Singh A, Patel T, Hertel J, et al. Safety of ferumoxytol in patients with anemia and CKD. Am J Kidney Dis. 2008;52(5):907-915.

100. Wu CY, Pu Y, Liu G, et al. MR imaging of human pancreatic cancer xenograft labelled with superparagmagnetic iron oxide in nude mice. Contrast Media Mol Imaging. 2012;7(1):51-58.

101. Laurent S, Dutz S, Hafeli UO, et al. Magnetic fluid hyperthermia: Focus on super paramagnetic iron oxide nanoparticles. Adv Colloid Interface Sci. 2011;166(1-2):8-23.

102. Kossatz S, Ludwig R, Dahring H, et al. High therapeutic efficiency of magnetic hyperthermia in xenograft models achieved with moderate temperature dosages in the tumor area. Pharm Res DOI 10.1007/ s11095-014-1417-0. 2014

103. Basel MT, Balvida S, Wang $H$, et al. Cell-delivered magnetic nanoparticles caused hyperthermia-mediated increased survival in murine pancreatic cancer model. Int J Nanomedicine. 2012;7:297-306.

104. Johannsen M, Thiesen B, WustP, etal. Magnetic nanoparticlehyperthermia for prostate cancer. Int J Hyperthermia. 2010;26(8):790-795.

105. West DL, White SB, Zhang Z, et al. Assessment and optimization of electroporation-assisted tumoral nanoparticle uptake in a nude mouse model of pancreatic ductal adenocarcinoma. Int $J$ Nanomedicine. 2014;9:4169-4176.

106. Hedgire SS, Mino-Kenudson M, Elmi A, et al. Enhanced primary tumor delineation in pancreatic adenocarcinoma using ultra small super paramagnetic iron oxide nanoparticle-ferumoxytol: an initial experience with histopathological correlation. Int $J$ Nanomedicine. 2014;9:1891-1896. 
107. Yallapu MM, Ebeling MC, Khan S, et al. Novel curcmin-loaded magnetic nanoparticles for pancreatic cancer treatment. Mol Cancer Ther. 2013;12(8):1471-1480.

108. Jain TK, Morales MA, Sahoo SK, et al. Iron oxide nanoparticles for sustained delivery of anticancer agents. Mol Pharm. 2005;2(3):194-205.

109. Vittorio O, Voliani V, Faraci P, et al. Magnetic catechin-dextran conjugate as targeted therapeutic for pancreatic tumor cells. J Drug Target. 2014;22(5):408-415.

110. Hwu JR, Lin YS, Josephrajan T, et al.Targeted paclitaxel by conjugation to iron oxide and gold nanoparticles. J Am Chem Soc. 2009;131(1):66-68.

111. Kievit FM, Zhang M. Surface engineering of iron oxide nanoparticles for targeted cancer therapy. Acc Chem Res. 2011;44(10):853-862.

112. Lee GY, Qian WP, Wang L, et al. Theranostic nanoparticles with controlled release of gemcitabine for targeted therapy and MRI of pancreatic cancer. ACS Nano. 2013;7(3):2078-2089.

113. Barnett C, Gueorguieva M, Lees M, et al. Physical stability, biocompatibility and potential use of hybrid iron oxide-gold nanoparticles as drug carriers. J Nano Res. 2013;15:1076.

114. Lancaster DL, Patel N, Lennard L, et al. 6-Thioguanine in children with acute lymphoblastic leukaemia: influence of food on parent drug pharmacokinetics and 6-thioguanine nucleotide concentrations. $\mathrm{BrJ}$ Clin Pharmacol. 2001;51(6):531-539.

115. Zaza G, Cheok M, Krynetskaia N, et al. Thiopurine pathway. Pharmacogenet Genomics. 2010;20:573-574.

116. Wagstaff AJ, Brown SD, Holden MR, et al. Cisplatin drug delivery using gold-coated iron oxide nanoparticles for enhanced tumor targeting with external magnetic fields. Inorganica Chimica Acta. 2012;393:328-333.

117. Jain PK, El-Sayed IH, El-Sayed MA. Au nanoparticles target cancer. Nanotoday. 2007;2:18-29.

118. Pissuwan D, Valenzuela SM, Cortie MB. Therapeutic possibilities of plasmonically heated gold nanoparticles. Trends Biotechnol. 2006;24(2):62-67.

119. Rai P, Mallidi S, Zheng X, et al. Development and applications of photo-triggered theranostic agents. Adv Drug Deliver Rev. 2010;62(11):1094-1124.
120. Hirsch LR, Stafford RJ, Bankson JA, et al. P Natl Acad Sci USA100. 2003;13549-13554.

121. Guo Y, Zhang Z, Kim DH, et al. Photothermal ablation of pancreatic cancer cells with hybrid iron-oxide core gold-shell nanoparticles. Int $J$ Nanomedicine. 2013;8:3437-3446.

122. Ranade VV, Holliger MA. Drug Delivery Systems. Boca Raton FA CRC. 1996 ,

123. Gregoriadis G. Drug entrapment in liposomes. FEBS Lett. 1973;36(3):292-296.

124. Sinko PJ. Martin's Physical Pharmacy and Pharmaceutical Sciences (5th edn), Lippincott Williams \& Wilkins, Philadelphia. 2005,

125. Nii T, Ishii F. Encapsulation efficiency of water-soluble and insoluble drugs in liposomes prepared by the microencapsulation vesicle method. Int J Pharm. 2005;298(1):198-205.

126. El-Ridy MS, Mostafa DM, Shehab A, et al. Biological evaluation of pyrazinamide liposomes for treatment of Mycobacterium tuberculosis. Int J Pharm. 2007;330(1-2):82-88.

127. McNeil SE, Perrie Y. Gene delivery using cationic liposomes. Expert Opin Ther Pat. 2006;16(10):1371-1382.

128. MohammedAR, Coombes AGA, Perrie Y.Amino acids as cryoprotectants for liposomal delivery systems. Eur J Pharm Sci. 2007;30(5):406-413.

129. Mohammed AR, Weston N, Coombes AGA, et al. Liposome formulation of poorly water soluble drugs: optimisation of drug loading and SEM analysis of stability. I J Pharm. 2004;285(1-2):23-34.

130. Deng L, Ke X, He Z, et al. A MSLN-targeted multifunctional nanoimmunoliposome for MRI and targeting therapy in pancreatic cancer. Int J Nanomedicine. 2012;7:5053-5065.

131. Barnett CM, Lees MR, Curtis ADM, et al. Poly(allylamine) magnetomicelles for image guided drug delivery. Pharmaceut Nanotech. 2013;1(3):224-238.

132. Clare H, Lin PK, Tetley L, et al. The use of nano polymeric selfassemblies based on novel amphiphilic polymers for oral hydrophobic drug delivery. Pharm Res. 2012;29(3):782-794. 